\title{
ROBUST NONLINEAR CONTROL FOR PATH TRACKING OF A QUAD-ROTOR HELICOPTER
}

\author{
Guilherme V. Raffo, Manuel G. Ortega, and Francisco R. Rubio
}

\begin{abstract}
This paper presents a nonlinear robust control strategy to solve the path tracking problem for a quadrotor unmanned aerial vehicle. The main objective is to design controllers that provide certain required performances during the quadrotor flight, such as null tracking error and robustness in the presence of sustained external disturbances affecting the six degrees of freedom, parametric uncertainties, and unmodeled dynamics. The control structure is performed through a nonlinear $\mathscr{H}_{\infty}$ controller to stabilize the rotational movements and a control law based on the backstepping approach with integral action to track the reference trajectory. Simulation results are carried out to corroborate the effectiveness and the robustness of the proposed strategy.
\end{abstract}

Key Words: $\quad$ Nonlinear $\mathscr{H}_{\infty_{\infty}}$ control, integral backstepping approach, robust control, path tracking, unmanned aerial vehicle.

\section{INTRODUCTION}

Unmanned aerial vehicles (UAVs) constitute an area that has motivated the control community during recent years. Many areas of control and robotics, such as fusion sensors, computer vision techniques, state estimators, and control methodologies have been exploited to improve the performance of these kinds of systems.

Due to the electromechanical design of aerial vehicles, most of these systems are underactuated mechanical systems, i.e., they have fewer control inputs than degrees of freedom. Generally, the intentional design results in a weight and cost reduction of the vehicle. However, underactuated systems bring a complexity and an increased challenge to the control area. Techniques developed for fully actuated robots cannot be directly applied to these kind of mechanical systems, since most of the underactuated systems are not fully feedback linearizable and exhibit nonholonomic constraints [9]. Therefore, nonlinear modelling techniques and modern nonlinear control theory are often employed to achieve autonomous flight with high performance [5,3]. Usually, the control law design for underactuated systems considers only the dynamics of controlled degrees-of-freedom (DOF) into the

Manuscript received June 21, 2012; revised May 19, 2013; accepted September 24, 2013.

G. V. Raffo was with the Departamento de Ingeniera de Sistemas y Automática, Universidad de Sevilla. He is now with the Departamento de Engenharia Eletrônica, Universidade Federal de Minas Gerais, 31270-901, Belo Horizonte, MG, Brazil (corresponding author, e-mail: raffo@ufmg.br).

M. G. Ortega and F. R. Rubio are with the Departamento de Ingeniera de Sistemas y Automática, Universidad de Sevilla, 41092, Sevilla, Spain (email: \{mortega, rubio\}@us.es).

The authors would like to thank MCeI-FEDER for funding this work under grants DPI2010-19154 and DPI2012-37580-C02-02. error state vector and those remaining must be assumed to have stable zero dynamics, or they must be controlled in an outer loop.

This paper deals with a UAV in the quadrotor helicopter configuration, which presents some advantages when compared to a classic helicopter. This is mainly because it is lifted and propelled by four rotors, which makes it possible to reduce each individual rotor size and to maintain the total load capacity, when compared with a helicopter with one main rotor. Moreover, these vehicles do not require mechanical linkages to act on propellers. This reduces the design, maintenance, and cost of the vehicle. These facts, added to its high maneuverability, allow take-offs and landings, as well as flight in touch environment [12].

Since the last decade, many efforts have been made to control quadrotor helicopters and several strategies have been developed to tackle with the path tracking problem for this type of system. As the quadrotor helicopter configuration is constituted by four coplanar propellers (see Fig. 1), it is not static feedback exact linearizable for the desired controlled outputs, $x, y, z$ and $\psi$, i.e., the translational and yaw angle positions [19]. Therefore, two types of approaches are often used to perform path tracking of the quadrotor helicopter: on one hand, considering the output vector as $x, y, z$ and $\psi$, it is possible to use an augmented state vector with a double integrator of the thrust (the altitude control input), which generates coupling between translational and rotational motion allowing using the feedback linearization technique. This makes the system with the controlled outputs $x, y, z$ and $\psi$ output controllable $[19,20]$.

On the other hand, taking into account the quadrotor helicopter model split up into two subsystems, the rotational and the translational or, in some cases, the actuated and the unactuated, the path tracking can be performed by using 


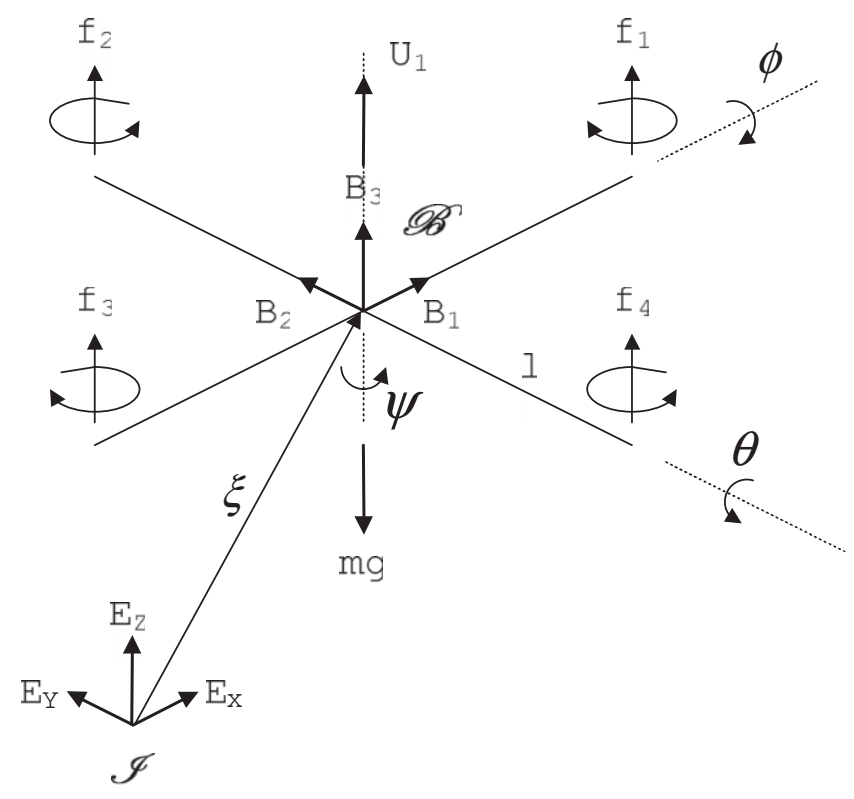

Fig. 1. Quadrotor helicopter scheme.

cascade control strategies. This approach uses an inner control loop for the rotational subsystem, or for the actuated degrees of freedom, combined with an outer loop to control the translational movements, which are needed to ensure the stability of the whole system. Control systems using this strategy can be found in $[6,2]$. This second approach is the one selected to develop the control strategy in this work.

One of the most commonly used control techniques for the quadrotor helicopter using cascade structures is the backstepping approach, which is applied to perform both path tracking and stabilization problems. Backstepping approaches applied to the quadrotor helicopter are found in $[17,16,15,11,23]$.

However, many control strategies tested on the quadrotor helicopter do not consider sustained disturbances on the six degrees of freedom, parametric and structural model uncertainties. In recent years, researchers considered them during the controller design. In some papers the quadrotor helicopter is controlled using a linear $\mathscr{H}_{\infty}$ controller based on linearized models [6].

Furthermore, robust sliding mode and backstepping approaches have also been developed. To improve the quadrotor helicopter path tracking performance when maintained winds disturb the system, a backstepping approach using integral action in the first step of the procedure is used in $[1,2,18,7]$. Xu and Özgüner propose an approach using sliding mode control for underactuated mechanical systems to stabilize a quadrotor helicopter when $30 \%$ uncertainty is added in each parameter of the model [28]. In [8] a backstepping approach is used to control the quadrotor helicopter, by applying backstepping on the Lagrangian form of the dynamics. Neural networks are introduced to estimate the aerodynamic components. In [21] a fractional order sliding mode controller is used to control a quadrotor helicopter taking into account the effect of the battery power loss.

In this paper, a nonlinear robust control strategy to solve the path tracking problem of the quadrotor helicopter is proposed. The main objective is to design controllers that provide certain required performances during the quadrotor flight, as null tracking error and robustness in presence of sustained external disturbances affecting the six degrees of freedom, parametric uncertainties and unmodeled dynamics. A nonlinear $\mathscr{H}_{\infty}$ controller is synthesized to stabilize the rotational movements, whereas a control law based on a backstepping approach with integral action is used to track the reference trajectory. In both controllers the integral action is considered, allowing the achievement of a null steady-state error when the system is disturbed. In the translational controller design, the integral backstepping procedure is used considering the integral term in its second step. This backstepping controller guarantees stability and convergence of the tracking error for a generic plant when a maintained disturbance affects the system and the reference signal is time-varying. On the other hand, one advantage of using the nonlinear $\mathscr{H}_{\infty}$ controller, compared with the linear one, to control of the rotational movements is related to the attraction basin, which is enlarged by the nonlinear approach.

The goal of the nonlinear $\mathscr{H}_{\infty}$ control theory is to achieve a bounded ratio between the energy of the so-called error signals and the energy of the disturbance signals. The nonlinear $\mathscr{H}_{6}$ approach considers Hamilton-Jacobi-BellmanIsaacs partial derivative equations (HJBI PDEs), which replace the Riccati equations in the case of the linear $\mathscr{H}_{\infty}$ control formulation. The main problem in the nonlinear case is the absence of a general method to solve these HJBI PDEs. Therefore, solutions have to be found for each particular case. In [22] a strategy to control fully actuated mechanical systems considering the tracking error dynamic equation is proposed. In such strategy a nonlinear $\mathscr{H}_{\infty}$ control, formulated via game theory, is applied. This strategy provides, through an analytical solution, a constant gain similar to the results obtained with the feedback linearization procedures.

The use of integral action in the backstepping technique was first proposed by [13]. The most common way to include integral action in this approach is to use parameter adaptation [14]. An analysis of different techniques using integral action in the backstepping approach is carried out by [25], where another two methods that consist in the augmentation of the the system dynamic with the integral state are presented.

The remainder of the paper is organized as follows. In Section II, a description of the quadrotor helicopter modeling is given. The control strategy proposed in this work is presented in Section III, where the backstepping control with 
integral action for the translational movements is developed and the nonlinear $\mathscr{H}_{\infty}$ controller for the rotational subsystem is presented. Some simulation results are shown in Section IV. Finally, the major conclusions of the paper are drawn in Section V.

\section{SYSTEM MODELING}

\subsection{Description}

The autonomous aerial vehicle used in this paper is a miniature quadrotor helicopter. The movement of the UAV results from changes on the lift force caused by adjusting the rotational velocities of the rotors. In order to achieve forward motion the velocity of the rear rotor must be increased and, simultaneously, the front rotor velocity must be decreased. The lateral displacement is performed with the same procedure but using the right and left propellers. Yaw movement is obtained from the difference in the counter-torque between each pair of propellers, i.e., accelerating the two clockwise turning rotors while decelerating the counter-clockwise turning rotors, and vice-versa.

The dynamic model of the system is obtained under the assumption that the vehicle is a rigid body in the space subject to one main force (thrust) and three torques. Therefore, the helicopter is considered an underactuated mechanical system with six degrees of freedom and only four control inputs. Since this kind of system is a flight vehicle of lightweight structure, gyroscopic effects resulting from the rotation of the rigid body and the four propellers should be included in the dynamic model. Moreover, assuming a realistic hypothesis that the center of mass is displaced by a position $r$ from the center of rotation, a strongly-coupled dynamic model should be considered. Due to already present complexities, some assumptions are made in the model for control purposes. Firstly, the gyroscopic effects caused by the propellers will be considered as unknown, because it is assumed, at the motion control design stage, that there is no access to the rotor speed. Consequently, these effects will be neglected for the control design and will be considered as external disturbances. Furthermore, to overcome the strong coupling between rotational and translational movements, the center of mass and the body-fixed frame origin are assumed congruent, which leads to a decentralized dynamic model allowing to design cascade control strategies. The ground effect is also neglected and, for controller synthesis purposes, the helicopter structure is assumed to be symmetric, which results in a moment of inertia tensor with just diagonal inertia terms. Therefore, all mentioned aerodynamic effects are neglected and assumed as uncertainties in the control design stage, and most of them taken into account in the model used to emulate the quadrotor helicopter model.

\subsection{Helicopter dynamics}

The helicopter as a rigid body is characterized by a frame linked to it. Let $\mathscr{B}=\left\{B_{1}, B_{2}, B_{3}\right\}$ be the frame fixed to the body, where the $B_{1}$ axis points towards the front of the vehicle, $B_{2}$ is orthogonal to $B_{1}$ and negative to starboard in the horizontal plane, whereas $B_{3}$ is oriented in the ascendant sense and orthogonal to the plane $B_{1} O B_{2}$. The inertial frame $\mathscr{T}=\left\{E_{x}, E_{y}, E_{z}\right\}$ is considered fixed with respect to the earth (see Fig. 1).

The vector $\xi=\left[\begin{array}{lll}x & y & z\end{array}\right]^{\prime}$ represents the position of the helicopter mass center expressed in the inertial frame $\mathscr{T}^{*}$ The vehicle orientation is given by a rotation matrix $\boldsymbol{R}_{\mathscr{T}}: \mathscr{B} \rightarrow \mathscr{T}$, where $\boldsymbol{R}_{\mathscr{T}} \in S O(3)$ is an orthonormal rotation matrix [9]. In this work the XYZ Euler angles, $\eta=\left[\begin{array}{lll}\varphi & \theta & \psi\end{array}\right]^{\prime} \in \mathfrak{R}^{3}$, are used to describe the helicopter rotation in the three-Euclidean space with respect to the bodyfixed frame. For a more detailed description of the quadrotor helicopter modeling see [24].

The helicopter equations of motion can be obtained by the Euler-Lagrange formalism based on the kinetic and potential energy concept, which compose the Lagrangian of the helicopter model, $\mathscr{L}(\boldsymbol{q}, \dot{\boldsymbol{q}})$. The generalized coordinate vector is given by $\boldsymbol{q}=\left[\begin{array}{ll}\xi^{\prime} & \eta^{\prime}\end{array}\right]^{\prime} \in \mathfrak{R}^{6}$.

Based on the assumption that the helicopter center of mass is congruent with the center of rotation, the Lagrangian does not contain kinetic energy terms combining $\dot{\xi}$ and $\dot{\eta}$. Consequently, the Euler-Lagrange equations can be divided into translational and rotational dynamics (see Fig. 2; Quadrotor Helicopter block). The translational movement can be expressed by the following equation [23]:

$$
m \ddot{\boldsymbol{\xi}}+m g \boldsymbol{e}_{3}=\boldsymbol{f}_{\xi},
$$

where $\boldsymbol{f}_{\boldsymbol{\xi}}=\boldsymbol{R}_{J} \hat{\boldsymbol{f}}+\boldsymbol{\alpha}_{T}$ is the translational force applied to the helicopter due to the main control input $U_{1}$ in $z$ axis direction, with $\boldsymbol{R}_{\mathscr{J}} \hat{\boldsymbol{f}}=\boldsymbol{R}_{\mathscr{T}_{e 3}} U_{1}$, and $\boldsymbol{\alpha}_{T}=\left[\begin{array}{lll}A_{x} & A_{y} & A_{z}\end{array}\right]^{\prime}$ is the aerodynamic force vector, whose components are in the $E_{x}, E_{y}$ and $E_{z}$ axes, respectively (The notation $\boldsymbol{e}_{3}$ represents the vector $\boldsymbol{e}_{3}=\left[\begin{array}{lll}0 & 0 & 1\end{array}\right]^{\prime}$. Thus, the therm $\boldsymbol{R}_{\mathscr{T} 3}$ denotes the third column of the rotation matrix.) Aerodynamic forces and moments are considered as external disturbances in the controller design.

Equation (1) can be expressed by means of state vector $\xi$, yielding:

$$
\left\{\begin{array}{l}
\ddot{x}=\frac{1}{m}(\cos \psi \sin \theta \cos \phi+\sin \psi \sin \phi) U_{1}+\frac{A_{x}}{m} \\
\ddot{y}=\frac{1}{m}(\sin \psi \sin \theta \cos \phi-\cos \psi \sin \phi) U_{1}+\frac{A_{y}}{m}, \\
\ddot{z}=-g+\frac{1}{m}(\cos \theta \cos \phi) U_{1}+\frac{A_{z}}{m}
\end{array}\right.
$$

\footnotetext{
*The prime notation ' denotes transpose.
} 


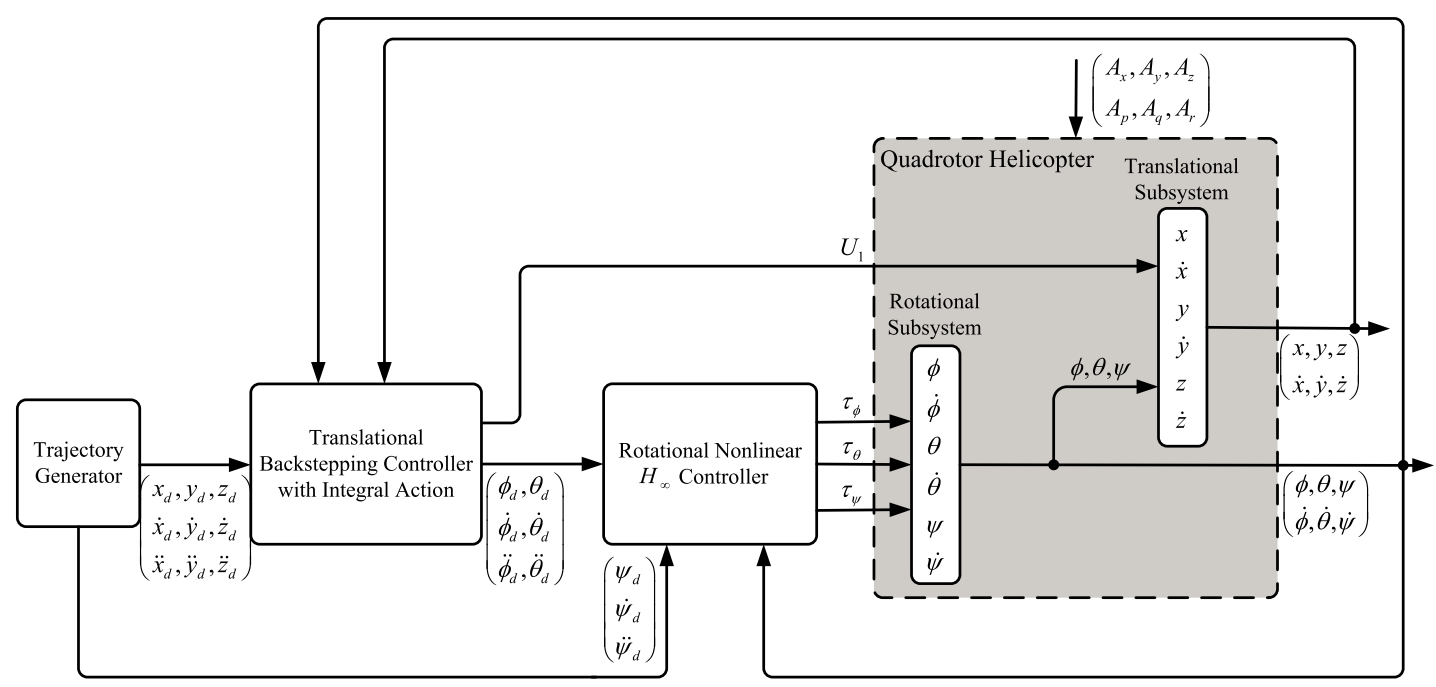

Fig. 2. Quadrotor helicopter control structure.

where $m$ is the helicopter mass and $g$ is the gravitational acceleration.

The equations of the rotational motion are written as a function of the generalized coordinate $\boldsymbol{\eta}$. Let $\boldsymbol{W}_{\boldsymbol{\eta}}$ be the Euler matrix, which relates the time-derivative of the Euler angles, $\dot{\eta}$, with the angular velocities expressed in the body-fixed frame, $\omega$, and is given by:

$$
\boldsymbol{W}_{\eta}=\left[\begin{array}{ccc}
1 & 0 & -\sin \theta \\
0 & \cos \phi & \sin \phi \cos \theta \\
0 & -\sin \phi & \cos \phi \cos \theta
\end{array}\right],
$$

the inertia matrix is defined by $\boldsymbol{M}(\boldsymbol{\eta})=\boldsymbol{W}_{\eta}^{\prime} \boldsymbol{J} \boldsymbol{W}_{\eta}$, where $\boldsymbol{J}=\operatorname{diag}\left(I_{x x}, I_{y y}, I_{z z}\right)$ is the diagonal moment of inertia tensor. Then the Euler-Lagrange rotational equations in terms of $\boldsymbol{\eta}$ can be written, in a general form, as follows [4]:

$$
M(\eta) \ddot{\eta}+C(\eta, \dot{\eta}) \dot{\eta}=\tau_{\eta_{a}}+\tau_{\eta_{d}}
$$

where $\tau_{\eta_{a}}$ is the applied roll, pitch and yaw control torque vector, and $\tau_{\eta_{d}}=\left[\begin{array}{lll}A_{p} & A_{q} & A_{r}\end{array}\right]^{\prime}$ is the aerodynamic torque vector. The Coriolis and centrifugal force matrix $\boldsymbol{C}(\boldsymbol{\eta}, \dot{\boldsymbol{\eta}})$ is not unique, but the vector $\boldsymbol{C}(\boldsymbol{\eta}, \dot{\boldsymbol{\eta}}) \dot{\boldsymbol{\eta}}$ is indeed unique. For the sake of convenience, in this work this matrix is obtained through the well-known Christoffel Symbols (of the first kind), $c_{i j k}(\boldsymbol{\eta})$, defined as follows [26]:

$$
c_{i j k}:=\frac{1}{2}\left\{\frac{\partial m_{k j}}{\partial \eta_{i}}+\frac{\partial m_{k i}}{\partial \eta_{j}}-\frac{\partial m_{i j}}{\partial \eta_{k}}\right\}
$$

and the $(k, j)$ th element of the matrix $\boldsymbol{C}(\boldsymbol{\eta}, \dot{\boldsymbol{\eta}})$ is given by:

$$
\begin{aligned}
c_{k j} & =\sum_{i=1}^{3} c_{i j k}(\eta) \dot{\eta}_{i} \\
& =\sum_{i=1}^{3} \frac{1}{2}\left\{\frac{\partial m_{k j}}{\partial \eta_{i}}+\frac{\partial m_{k i}}{\partial \eta_{j}}-\frac{\partial m_{i j}}{\partial \eta_{k}}\right\} \dot{\eta}_{i} .
\end{aligned}
$$

\section{THE PROPOSED CONTROL STRATEGY}

The control strategy used in this work is based on the decentralized structure of the quadrotor helicopter model that is composed by the dynamic equations (1) and (4) (see Fig. 2).

The goal of the strategy is to reach a robust flight of the quadrotor helicopter in the presence of sustained external disturbances, parametric uncertainties and unmodelled dynamics. To achieve it, two nonlinear techniques are combined.

Initially, the reference trajectory for the translational movements is provided off-line by the Trajectory Generator block. The computation of this trajectory is based on a virtual reference vehicle whose model is the same as the QuadRotor simplified one for the translational motion. Thereby, starting from a desired route for the translational movements, $x_{d}, y_{d}, z_{d}$ and their derivatives are computed. The reference yaw angle is defined separately. This trajectory is generated under the following assumptions: there are no external disturbances acting on the virtual vehicle; and the attitude of the virtual vehicle is assumed stabilized.

For the inner-loop control, a nonlinear $\mathscr{H}_{\infty}$ controller for the rotational subsystem is used to perform the QuadRotor helicopter stabilization. The angular position and velocity are controlled in this loop, being the torques applied 
on the three axis, $\tau_{\eta_{a}}=\left[\begin{array}{lll}\tau_{\phi_{a}} & \tau_{\theta_{a}} & \tau_{\psi_{a}}\end{array}\right]^{\prime}$, the manipulated variables. To obtain null steady-state error in presence of sustained external disturbances, the integral of the angular position error is considered. Due to the cascade structure of this strategy and taking into account the closed-loop performance achieved by the nonlinear $\mathscr{H}_{\infty}$ inner-loop controller, the Euler angles can be considered as time-varying parameters on the design of the translational controllers.

To obtain a robust path tracking, an integral backstepping approach is used to design the quadrotor translational motion controller. As can be seen in [23], the standard backstepping procedure is not able to perform the tracking task, without considering the integral action, when sustained disturbances affect the system. Therefore, an integral action is added on the control design. Moreover, in the backstepping approach presented in this work the integral action is considered in its second step, which guarantees stability and convergence of the tracking error for a generic plant when a maintained disturbance affect the system and the reference signal is time-varying. This same benefit is not obtained when the integral term is considered in the first step of the backstepping control design. Control strategies applied to the quadrotor helicopter using the backstepping approach with integral action in its first step are found in $[2,18]$.

The translational motion control is performed in two stages. In the first one, the altitude, $z$, is controlled and the desired total thrust, $U_{1}$, is the manipulated signal. In the second stage, the reference of pitch and roll angles $\left(\theta_{d}\right.$ and $\phi_{d}$, respectively) are generated through two virtual inputs, computed to follow the desired $x y$ movement. In this step, the control variable $U_{1}$ is used as a time-varying parameter.

The development of each one of these controllers is analyzed in the following.

\subsection{Backstepping control with integral action for path tracking}

In this section a control law to solve the path tracking problem by translational movements is designed. Backstepping technique can be used to design this control law in such a way that the subsystem is forced to track the reference trajectory.

However, a helicopter can be subject to external disturbances like wind gusts or, in the worst case, sustained winds. So that the integral action is considered in the backstepping approach. As a well known fact, the inclusion of the integral action allows the removal of constant steady-state offsets in closed-loop, besides to enable the path tracking in presence of sustained disturbances, unmodeled dynamics, and parameter deviations.

\subsubsection{Backstepping control with integral action formulation}

Consider a nonlinear system given by:

$$
\begin{aligned}
& \dot{\boldsymbol{x}}_{\mathbf{1}}=f_{1}\left(\boldsymbol{x}_{\mathbf{1}}\right)+g_{1}\left(\boldsymbol{x}_{\mathbf{1}}\right) \cdot \boldsymbol{x}_{\mathbf{2}} \\
& \dot{\boldsymbol{x}}_{\mathbf{2}}=f_{2}\left(\boldsymbol{x}_{\mathbf{1}}, \boldsymbol{x}_{\mathbf{2}}\right)+g_{2}\left(\boldsymbol{x}_{\mathbf{1}}, \boldsymbol{x}_{\mathbf{2}}\right) \cdot \boldsymbol{u}+\boldsymbol{d},
\end{aligned}
$$

where $\boldsymbol{x}_{\mathbf{1}}$ and $\boldsymbol{x}_{\mathbf{2}}$ are the state vectors, $\boldsymbol{u}$ is the control input vector and $\boldsymbol{d}$ is an unknown sustained disturbance vector.

First of all, the backstepping state transformation is considered:

$$
\begin{aligned}
& e_{1}=x_{1}-x_{d}(t) \\
& e_{2}=x_{2}-\alpha\left(e_{1}, t\right),
\end{aligned}
$$

where $\alpha\left(\boldsymbol{e}_{1}, t\right)$ is a virtual control and $\boldsymbol{x}_{d}(t)$ is a reference signal. The goal of this approach is to solve the tracking problem $\lim _{t \rightarrow \infty} \boldsymbol{e}_{1}(t)=0$.

The integral term $\boldsymbol{\xi}(t)=\int_{0}^{t} \boldsymbol{e}_{2}(\tau) d \tau$ is considered in the second step of the backstepping approach. As commented in [25], this method guarantees convergence for constant or time-varying reference signals, which could not be guaranteed for a generic plant when the integral action is added in the first step of this control design.

Then, for the plant (7), the system for the first step is given by:

$$
\begin{aligned}
\dot{\boldsymbol{e}}_{1}= & f_{1}\left(\boldsymbol{e}_{1}+\boldsymbol{x}_{d}(t)\right)+g_{1}\left(\boldsymbol{e}_{1}+\boldsymbol{x}_{d}(t)\right) \cdot \boldsymbol{e}_{2} \\
& +g_{1}\left(\boldsymbol{e}_{1}+\boldsymbol{x}_{d}(t)\right) \cdot \boldsymbol{\alpha}\left(\boldsymbol{e}_{1}, t\right)-\dot{\boldsymbol{x}}_{d}(t) .
\end{aligned}
$$

where functions $f_{1}$ and $g_{1}$ are written with respect to the error variables (8).

A first positive definite Lyapunov function $V_{1}\left(\boldsymbol{e}_{1}, t\right)$ is chosen as follows:

$$
V_{1}\left(\boldsymbol{e}_{1}, t\right)=\frac{1}{2} \boldsymbol{e}_{1}^{\prime} \cdot \boldsymbol{e}_{1}
$$

Its time derivative becomes

$$
\dot{V}_{1}\left(\boldsymbol{e}_{1}, t\right)=\boldsymbol{e}_{1}^{\prime} \cdot \dot{\boldsymbol{e}}_{1}
$$

By substituting (9) in (11), the virtual control, $\boldsymbol{\alpha}\left(\boldsymbol{e}_{\mathbf{1}}, t\right)$, is defined to guarantee that the time derivative of the candidate Lyapunov function is negative definite when $\boldsymbol{e}_{2}=0$. It is given as follows:

$$
\alpha\left(e_{1}, t\right)=g_{1}\left(e_{1}+x_{d}(t)\right)^{-1} \cdot\left[-f_{1}\left(e_{1}+x_{d}(t)\right)-C_{1} \cdot e_{1}+\dot{x}_{d}(t)\right],
$$

where $\boldsymbol{C}_{\mathbf{1}}=\boldsymbol{C}_{\mathbf{1}}^{\prime}>0$. This virtual control law cancels all nonlinear terms of (9) and depends only on $\boldsymbol{e}_{1}$ and the reference signal.

Consequently, the time derivative of the Lyapunov function can be written as:

$$
\dot{V}_{1}\left(e_{1}, t\right)=-e_{1}^{\prime} \cdot C_{1} \cdot e_{1}+e_{1}^{\prime} \cdot g_{1}\left(e_{1}+x_{d}(t)\right) \cdot e_{2}
$$

allowing the control design to proceed, which for $\boldsymbol{e}_{2}=0$ it is negative definite. 
In the second step the integral action is introduced and the following system is considered:

$$
\begin{aligned}
\dot{\xi}= & \boldsymbol{e}_{2} \\
\dot{\boldsymbol{e}}_{2}= & f_{2}\left(\boldsymbol{e}_{1}+\boldsymbol{x}_{d}(t), \boldsymbol{e}_{2}+\boldsymbol{\alpha}\left(\boldsymbol{e}_{1}, t\right)\right)+g_{2}\left(\boldsymbol{e}_{1}+\boldsymbol{x}_{d}(t), \boldsymbol{e}_{2}\right. \\
& \left.+\boldsymbol{\alpha}\left(\boldsymbol{e}_{1}, t\right)\right) \cdot \boldsymbol{u}-\boldsymbol{\alpha}\left(\boldsymbol{e}_{1}, t\right)+\boldsymbol{d},
\end{aligned}
$$

where functions $f_{2}$ and $g_{2}$ are written with respect to the error variables (8) and (from now on the arguments of the system functions will be omitted):

$$
\begin{aligned}
& \dot{\alpha}\left(\boldsymbol{e}_{1}, t\right)=\frac{\partial \alpha}{\partial \boldsymbol{e}_{1}} \cdot \dot{\boldsymbol{e}}_{1}+\frac{\partial \alpha}{\partial t} \\
& \frac{\partial \boldsymbol{\alpha}}{\partial \boldsymbol{e}_{1}}= g_{1}^{-1} \cdot\left[-g_{1} \cdot\left(-f_{1}-\boldsymbol{C}_{1} \cdot \boldsymbol{e}_{1}+\dot{\boldsymbol{x}}_{\boldsymbol{d}}(t)\right) \cdot D_{\left(\boldsymbol{e}_{1}+x_{d}(t)\right)} g_{1}\right. \\
&\left.-D_{\left(\boldsymbol{e}_{1}+\boldsymbol{x}_{d}(t)\right)} f_{1}-\boldsymbol{C}_{1}\right] \\
& \frac{\partial \alpha}{\partial t}= g_{1}^{-1} \cdot\left[-g_{1}\left(-f_{1}-C_{1} \boldsymbol{e}_{1}+\dot{\boldsymbol{x}}_{d}(t)\right) \cdot D_{\left(\boldsymbol{e}_{1}+x_{d}(t)\right)} g_{1} \cdot \dot{\boldsymbol{x}}_{\boldsymbol{d}}(t)\right. \\
&\left.-D_{\left(\boldsymbol{e}_{1}+\boldsymbol{x}_{d}(t)\right)} f_{1} \cdot \dot{\boldsymbol{x}}_{\boldsymbol{d}}(t)+\ddot{\boldsymbol{x}}_{\boldsymbol{d}}(t)\right] .
\end{aligned}
$$

A second Lyapunov function is chosen as:

$$
V_{2}\left(\boldsymbol{\xi}, \boldsymbol{e}_{1}, \boldsymbol{e}_{2}, t\right)=V_{1}+\frac{1}{2} \boldsymbol{\xi}^{\prime} \cdot \boldsymbol{K}_{\xi} \cdot \boldsymbol{\xi}+\frac{1}{2} \boldsymbol{e}_{2}^{\prime} \cdot \boldsymbol{e}_{2}
$$

where $\boldsymbol{K}_{\xi}=\boldsymbol{K}_{\xi}^{\prime}>0$, and its time derivative is given by:

$$
\begin{aligned}
\dot{V}_{2}\left(\boldsymbol{\xi}, \boldsymbol{e}_{1}, \boldsymbol{e}_{2}, t\right)= & \dot{V}_{1}\left(\boldsymbol{e}_{1}, t\right)+\boldsymbol{\xi}^{\prime} \cdot \boldsymbol{K}_{\xi} \cdot \dot{\boldsymbol{\xi}}+\boldsymbol{e}_{2}^{\prime} \cdot \dot{\boldsymbol{e}}_{2} \\
= & -\boldsymbol{e}_{1}^{\prime} \cdot \boldsymbol{C}_{1} \cdot \boldsymbol{e}_{1}+\boldsymbol{e}_{1}^{\prime} \cdot g_{1} \cdot \boldsymbol{e}_{2}+\boldsymbol{\xi}^{\prime} \cdot \boldsymbol{K}_{\xi} \cdot \boldsymbol{e}_{2} \\
& +\boldsymbol{e}_{2}^{\prime} \cdot\left[f_{2}+g_{2} \cdot \boldsymbol{u}-\dot{\alpha}\left(\boldsymbol{e}_{1}, t\right)+\boldsymbol{d}\right] .
\end{aligned}
$$

Thus, in order to guarantee that the time derivative of the candidate Lyapunov function, (17), is definite negative when no exogenous disturbances are acting on the system, the computed control law, $\boldsymbol{u}$, is defined as follows:

$$
\boldsymbol{u}=g_{2}^{-1} \cdot\left[-f_{2}+\dot{\alpha}\left(\boldsymbol{e}_{1}, t\right)-g_{1}^{\prime} \cdot \boldsymbol{e}_{1}-\boldsymbol{K}_{\xi} \cdot \boldsymbol{\xi}-\boldsymbol{C}_{2} \cdot \boldsymbol{e}_{2}\right],
$$

with $\boldsymbol{C}_{2}=\boldsymbol{C}_{2}^{\prime}>0$.

Replacing this control law in (17) yields to:

$$
\dot{V}_{2}\left(\xi, e_{1}, e_{2}, t\right)=-e_{1}^{\prime} \cdot C_{1} \cdot e_{1}-e_{2}^{\prime} \cdot C_{2} \cdot e_{2}+e_{2}^{\prime} d .
$$

which guarantees that the equilibrium $\left(\boldsymbol{\xi}, \boldsymbol{e}_{1}, \boldsymbol{e}_{2}\right)=0$ of the closed-loop with $\boldsymbol{d}=0$ is UGAS (uniform global asymptotic stable). If $\boldsymbol{d} \neq 0$, the new equilibrium $\left(\boldsymbol{\xi}, \boldsymbol{e}_{1}, \boldsymbol{e}_{2}\right)=\left(\boldsymbol{K}_{\xi}^{-1} \cdot \boldsymbol{d}, 0,0\right)$ of the closed loop is UGAS for any constant $\boldsymbol{d}$ (for more details see [25]).

Considering the backstepping state transformation (8) and the virtual control law (12), the control law (18) can be written in terms of a nonlinear PID with a feedforward action control law as follows:

$$
\begin{aligned}
\boldsymbol{u}= & -\boldsymbol{K}_{\boldsymbol{I}} \cdot \boldsymbol{\xi}-\boldsymbol{K}_{P} \cdot\left[\boldsymbol{x}_{1}-\boldsymbol{x}_{\boldsymbol{d}}(t)\right] \\
& -\boldsymbol{K}_{\boldsymbol{D}} \cdot\left[\boldsymbol{x}_{2}-g_{1}^{-1} \cdot\left(\dot{\boldsymbol{x}}_{\boldsymbol{d}}(t)-f_{1}\right)\right]+\boldsymbol{F}_{F},
\end{aligned}
$$

where

$$
\begin{aligned}
\boldsymbol{K}_{\boldsymbol{I}} & =g_{2}^{-1} \cdot \boldsymbol{K}_{\boldsymbol{\xi}}, \\
\boldsymbol{K}_{\boldsymbol{P}} & =g_{2}^{-1} \cdot\left(g_{1}^{\prime}+\boldsymbol{C}_{2} \cdot g_{1}^{-1} \cdot \boldsymbol{C}_{\mathbf{1}}\right), \\
\boldsymbol{K}_{\boldsymbol{D}} & =g_{2}^{-1} \cdot \boldsymbol{C}_{\mathbf{2}}, \\
\boldsymbol{F}_{\boldsymbol{F}} & =g_{2}^{-1} \cdot\left[-f_{2}+\dot{\boldsymbol{\alpha}}\left(\boldsymbol{e}_{\mathbf{1}}, t\right)\right] .
\end{aligned}
$$

\subsubsection{Translational subsystem backstepping control}

The controller for the translational subsystem is performed in two phases. The first one controls the helicopter height through the input $U_{1}$, whereas the second one makes use of this signal as a time variant parameter in the linear $x$ and $y$ motions to compute two virtual control inputs, $u_{x}^{c}$ and $u_{v}^{c}$

For the controller design, the system (2) can be written in a state-space form as $\overline{\boldsymbol{\xi}}=f\left(\overline{\boldsymbol{\xi}}, \boldsymbol{u}_{\boldsymbol{\xi}}\right)$, where $\bar{\xi}=\left[\begin{array}{llllllll}x & u_{0} & y & v_{0} & z & w_{0}\end{array}\right]^{\prime}$ stands for the state-space vector of the system, and $u_{0}, v_{0}$, and $w_{0}$ are the components of the linear velocity of the vehicle mass center expressed in the inertial frame.

From (2) and the new state-space vector, the system dynamic equation to control design can be written in the following form:

$$
\dot{\bar{\xi}}=f\left(\bar{\xi}, \boldsymbol{u}_{\xi}\right)=\left[\begin{array}{c}
u_{0} \\
u_{x} \frac{U_{1}}{m}+\frac{A_{x}}{m} \\
v_{0} \\
u_{y} \frac{U_{1}}{m}+\frac{A_{y}}{m} \\
w_{0} \\
-g+(\cos \phi \cos \theta) \frac{U_{1}}{m}+\frac{A_{z}}{m}
\end{array}\right],
$$

with:

$$
\begin{aligned}
& u_{x} \triangleq \cos \psi \sin \theta \cos \phi+\sin \psi \sin \phi \\
& u_{y} \triangleq \sin \psi \sin \theta \cos \phi-\cos \psi \sin \phi .
\end{aligned}
$$

Equations (22) show that the movement through the $x$ and $y$ axes depends on the control input $U_{1}$. In fact, $U_{1}$ is the designed total thrust magnitude to obtain the desired linear movement, while $u_{x}$ and $u_{y}$ can be considered as the directions of $U_{1}$ that cause the movement through the $x$ and $y$ axes, respectively.

In the following subsections the controllers based on the backstepping approach with integral action are developed. 
Altitude control. To design the altitude controller the last two equations of the system (22) with the unknown disturbance term $A_{z} \neq 0$ are considered. Due to the quadrotor helicopter cascade structure, the Euler angles are considered to be time-varying parameters.

The first step considers the backstepping state transformation:

$$
\begin{aligned}
& e_{1_{z}}=z-z_{d}(t) \\
& e_{2_{z}}=\dot{z}-\alpha_{z}\left(e_{1_{z}}, t\right),
\end{aligned}
$$

where the integral term is $\xi_{z}(t)=\int_{0}^{t} e_{z_{z}}(\tau) d \tau$, and the virtual control, $\alpha_{1}\left(e_{1_{z}}, t\right)$, is obtained from (12) for the altitude system as follows:

$$
\alpha_{z}\left(e_{1_{z}}, t\right)=-c_{1_{z}} e_{1_{z}}+\dot{z}_{d}(t)
$$

Then, the system based on the backstepping approach for altitude control is given by:

$$
\begin{aligned}
& \dot{e}_{1_{z}}=e_{2_{z}}+\alpha_{z}\left(e_{1_{z}}, t\right) \\
& \dot{\xi}_{z}=e_{2_{z}} \\
& \dot{e}_{2_{z}}=-g+(\cos \phi \cos \theta) \frac{U_{1}}{m}+\frac{A_{z}}{m}-\dot{\alpha}_{z}\left(e_{1_{z}}, t\right),
\end{aligned}
$$

where the thrust $U_{1}$, computed through the backstepping approach, is defined as follows:

$$
\begin{aligned}
U_{1}= & \frac{m}{\cos \phi \cos \theta} \cdot\left[-k_{\xi_{z}} \xi_{z}-\left(1+c_{2_{z}} c_{1_{z}}\right)\left(z-z_{d}\right)\right. \\
& \left.-\left(c_{1_{z}}+c_{2_{z}}\right)\left(\dot{z}-\dot{z}_{d}\right)+g+\ddot{z}_{d}\right] .
\end{aligned}
$$

Longitudinal and lateral movement control. To compute $u_{x}^{c}$ and $u_{y}^{c}$ the same backstepping approach is used and the following backstepping transformation is considered:

$$
\begin{aligned}
& e_{1_{x y}}=x_{1_{x y}}-x_{d x y}(t) \\
& e_{2 x y}=x_{2 x y}-\alpha_{x y}\left(e_{1 x y}, t\right),
\end{aligned}
$$

where:

$$
\boldsymbol{x}_{\mathbf{1}_{x y}}=\left[\begin{array}{c}
x \\
y
\end{array}\right], \quad \boldsymbol{x}_{2_{x y}}=\left[\begin{array}{c}
\dot{x} \\
\dot{y}
\end{array}\right], \quad \boldsymbol{\xi}_{x y}=\left[\begin{array}{l}
\int_{0}^{t} e_{2_{x}}(\tau) d \tau \\
\int_{0}^{t} e_{2_{y}}(\tau) d \tau
\end{array}\right],
$$

and the virtual control, $\boldsymbol{\alpha}_{x y}\left(\boldsymbol{e}_{1_{x y}}, t\right)=\left[\alpha_{x}\left(e_{1_{x}}, t\right) \quad \alpha_{y}\left(e_{1 y}, t\right)\right]^{\prime}$, is obtained from equation (12).

Therefore, the control law is given by:

$$
\begin{aligned}
{\left[\begin{array}{l}
u_{x}^{c} \\
u_{y}^{c}
\end{array}\right]=} & \frac{m}{U_{1}} \cdot\left[-\boldsymbol{K}_{\xi_{x y}} \boldsymbol{\xi}_{x y}-\left(\mathbb{1}+\boldsymbol{C}_{2_{x y}} \boldsymbol{C}_{\mathbf{1}_{x y}}\right)\left(\boldsymbol{x}_{\mathbf{1}_{x y}}-\boldsymbol{x}_{d_{x y}}\right)\right. \\
& \left.-\left(\boldsymbol{C}_{2_{x y}}+\boldsymbol{C}_{\mathbf{1}_{x y}}\right)\left(\boldsymbol{x}_{2_{x y}}-\dot{\boldsymbol{x}}_{d_{x y}}\right)+\ddot{\boldsymbol{x}}_{d_{x y}}\right],
\end{aligned}
$$

where $U_{1}$ is assumed non-zero and

$$
\boldsymbol{C}_{\mathbf{1}_{x y}}=\left[\begin{array}{cc}
c_{1_{x}} & 0 \\
0 & c_{1_{y}}
\end{array}\right], \boldsymbol{C}_{\boldsymbol{2}_{x y}}=\left[\begin{array}{cc}
c_{2_{x}} & 0 \\
0 & c_{2_{y}}
\end{array}\right], \boldsymbol{K}_{\xi_{\boldsymbol{y} y}}=\left[\begin{array}{cc}
k_{\xi_{x}} & 0 \\
0 & k_{\xi_{y}}
\end{array}\right] .
$$

Taking into account that the virtual control inputs $u_{x}^{c}$ and $u_{y}^{c}$ have been obtained to track the path reference in the $x y$ plane, the necessary values of $\phi$ and $\theta$ could be computed by (23). At this point, it should be noted that (23) constitutes a definition of the system to be controlled. Therefore, these values can not be set directly since these angles are two of the outputs of the rotational subsystem, being the nonlinear $\mathscr{H}_{\infty}$ inner-loop in charge of carrying out this task.

In consequence, a desired virtual direction vector, $\boldsymbol{u}_{x y}^{d}=\left[\begin{array}{ll}u_{x}^{d} & u_{y}^{d}\end{array}\right]^{\prime}$, must be defined in the same sense of (23) as follows:

$$
\begin{aligned}
& u_{y}^{d}(t)=\sin \psi(t) \sin \theta_{r}(t) \cos \phi_{r}(t)-\cos \psi(t) \sin \phi_{r}(t) . \\
& u_{y}^{d}(t)=\sin \psi(t) \sin \theta_{r}(t) \cos \phi_{r}(t)-\cos \psi(t) \sin \phi_{r}(t) .
\end{aligned}
$$

Thereby, by making the virtual control inputs equal to the desired values, $\boldsymbol{u}_{x y}^{c}=\boldsymbol{u}_{x y}^{\boldsymbol{d}}$, the reference of the roll and pitch angles, $\phi_{r}$ and $\theta_{r}$ respectively, are derived using (29). These references are necessary for the helicopter rotational loop.

\subsection{Rotational subsystem nonlinear $\mathscr{H}_{\infty}$ control}

In this section the rotational subsystem control law to achieve robustness in the presence of sustained disturbances, parametric uncertainties and unmodeled dynamics is synthesized. This control law is based on the full-actuated nonlinear $\mathscr{H}_{\infty}$ controller presented in [24], which is proposed to accomplish the required task.

The dynamic model of the rotational movements (4), obtained from the Euler-Lagrange formalism, is used in order to design the nonlinear $\mathscr{H}_{\infty}$ controller, which is carried out by a direct method.

As a first step to synthesize the control law, the tracking error vector is defined as follows:

$$
\tilde{\boldsymbol{x}}=\left[\begin{array}{c}
\dot{\tilde{\eta}} \\
\tilde{\boldsymbol{\eta}} \\
\int \tilde{\boldsymbol{\eta}} d t
\end{array}\right]=\left[\begin{array}{c}
\dot{\boldsymbol{\eta}}-\dot{\boldsymbol{\eta}}_{d} \\
\boldsymbol{\eta}-\boldsymbol{\eta}_{d} \\
\int\left(\boldsymbol{\eta}-\boldsymbol{\eta}_{d}\right) d t
\end{array}\right],
$$

where $\eta_{d}$ and $\dot{\eta}_{d} \in \mathfrak{R}^{n}$ are the desired trajectory and the corresponding velocity, respectively. Note that the integral 
term is considered in the error vector. This term will allow the achievement of a null steady-state error when sustained disturbances are acting on the system [24].

The following control law is proposed for the rotational subsystem:

$$
\begin{aligned}
\tau_{\eta_{a}=} & M(\eta) \ddot{\eta}+C(\eta, \dot{\eta}) \dot{\eta} \\
& -T_{1}^{-1}(\eta(\eta) T \dot{\tilde{x}}+C(\eta, \dot{\eta}) T \tilde{x})+T_{1}^{-1} u
\end{aligned}
$$

The proposed control law can be split up into three different parts: the first one consists of the first two terms of that equation, which are designed in order to compensate the system dynamics (4). The second part consists of terms including the error vector $\tilde{\boldsymbol{x}}$ and its time derivative, $\dot{\tilde{x}}$. Assuming $\tau_{\eta_{d}} \equiv 0$, these two terms of the control law enable perfect tracking, which means that they represent the essential control effort needed to perform the task. Finally, the third part includes a vector $\boldsymbol{u} \in \mathfrak{R}^{p}$, which represents the additional control effort needed for disturbance rejection.

It can also be pointed out that the previous control law might not seem a well posed system, however, it will be shown afterwards that the computed torque does not rely on joint accelerations, but on their references.

Matrix $\boldsymbol{T}$ in (31) can be partitioned as follows:

$$
T=\left[\begin{array}{lll}
T_{1} & T_{2} & T_{3}
\end{array}\right],
$$

with $\boldsymbol{T}_{1}=\rho \mathbb{1}$, where $\rho$ is a positive scalar, $\mathbb{1}$ is the $n$ th-order identity matrix, and $\boldsymbol{T}_{2}$ and $\boldsymbol{T}_{3}$ are $n$ th-order constant positive definite matrices.

Substituting the expression of the control law from (31) into the Euler-Lagrange equation of the system (4) and defining $\boldsymbol{d}=\boldsymbol{T}_{1} \tau_{\eta_{d}}$, the following expression is obtained:

$$
M(\eta) \boldsymbol{T} \dot{\tilde{x}}+C(\eta, \dot{\eta}) T \tilde{\boldsymbol{x}}=\boldsymbol{u}+\boldsymbol{d}
$$

where $\boldsymbol{d} \in \mathfrak{R}^{q}$ is the vector of external disturbances.

This expression represents the dynamic equation of the system error. Taking into account this nonlinear equation and the following cost variable $\zeta \in \mathfrak{R}^{(m+p)}$ :

$$
\zeta=W\left[\begin{array}{c}
h(x) \\
\boldsymbol{u}
\end{array}\right]
$$

where $h(\boldsymbol{x}) \in \mathfrak{R}^{m}$ represents a function of the error vector to be controlled and $\boldsymbol{W} \in \mathfrak{R}^{(m+p) \times(m+p)}$ is a weighting matrix, if the states $\boldsymbol{x}$ are assumed to be available for measurement, the nonlinear $\mathscr{H}_{\infty}$ problem can be posed as follows:

Find the smallest value $\gamma^{*} \geq 0$, such that, for any $\gamma \geq \gamma^{*}$ there exists a state feedback $\boldsymbol{u}=\boldsymbol{u}(\boldsymbol{x}, t)$, such that the $L_{2}$ gain from $\boldsymbol{d}$ to $\zeta$ is less than or equal to $\gamma$, that is:

$$
\int_{0}^{T}\|\zeta\|_{2}^{2} d t \leq \gamma^{2} \int_{0}^{T}\|\boldsymbol{d}\|_{2}^{2} d t
$$

where

$$
\|\boldsymbol{\zeta}\|_{2}^{2}=\boldsymbol{\zeta}^{\prime} \boldsymbol{\zeta}=\left[\begin{array}{ll}
h^{\prime}(\boldsymbol{x}) & \boldsymbol{u}^{\prime}
\end{array}\right] \boldsymbol{W}^{\prime} \boldsymbol{W}\left[\begin{array}{c}
h(\boldsymbol{x}) \\
\boldsymbol{u}
\end{array}\right]
$$

and the symmetric positive definite matrix $\boldsymbol{W}^{\prime} \boldsymbol{W}$ can be partitioned as follows:

$$
W^{\prime} W=\left[\begin{array}{cc}
Q & S \\
S^{\prime} & R
\end{array}\right]
$$

Matrices $\boldsymbol{Q}$ and $\boldsymbol{R}$ are symmetric positive definite and the fact that $\boldsymbol{W}^{\prime} \boldsymbol{W}>\mathbb{O}$ guarantees that $\boldsymbol{Q}-\boldsymbol{S} \boldsymbol{R}^{-1} \boldsymbol{S}^{\prime}>\mathbb{D}$.

Considering the definition of the vector error, $\tilde{\boldsymbol{x}}$, and the definition of the cost variable, $\zeta$, the following structures are considered for matrices $\boldsymbol{Q}$ and $\boldsymbol{S}$ in (35):

$$
Q=\left[\begin{array}{lll}
Q_{1} & Q_{12} & Q_{13} \\
Q_{12} & Q_{2} & Q_{23} \\
Q_{13} & Q_{23} & Q_{3}
\end{array}\right], \quad S=\left[\begin{array}{c}
S_{1} \\
S_{2} \\
S_{3}
\end{array}\right]
$$

To apply the theoretical results presented in [27], it is necessary to rewrite the nonlinear dynamic equation of the system error (32) into the standard form of the nonlinear $\mathscr{H}_{\infty}$ problem. This can be done by defining the following expressions:

$$
\begin{gathered}
\dot{\tilde{\boldsymbol{x}}}=f(\tilde{\boldsymbol{x}}, t)+g(\tilde{\boldsymbol{x}}, t) \boldsymbol{u}+k(\tilde{\boldsymbol{x}}, t) \boldsymbol{d}, \\
f(\tilde{\boldsymbol{x}}, t)=\boldsymbol{T}_{o}^{-1}\left[\begin{array}{ccc}
-\boldsymbol{M}^{-1} \boldsymbol{C} & \mathbb{1} & \mathbb{1} \\
\boldsymbol{T}_{1}^{-1} & \mathbb{1}-\boldsymbol{T}_{1}^{-1} \boldsymbol{T}_{2} & -1+\boldsymbol{T}_{1}^{-1}\left(\boldsymbol{T}_{2}-\boldsymbol{T}_{3}\right) \\
\mathbb{0} & \mathbb{1} & -\mathbb{1}
\end{array}\right] \boldsymbol{T}_{\boldsymbol{o}} \tilde{\boldsymbol{x}} \\
g(\tilde{\boldsymbol{x}}, t)=k(\tilde{\boldsymbol{x}}, t)=\boldsymbol{T}_{o}^{-1}\left[\begin{array}{c}
\boldsymbol{M}^{-1} \\
\mathbb{1} \\
\mathbb{0}
\end{array}\right],
\end{gathered}
$$

where $\mathbb{1}$ is the identity matrix, $\mathbb{D}$ the zero matrix, both of $n$th order, and:

$$
T_{o}=\left[\begin{array}{ccc}
T_{1} & T_{2} & T_{3} \\
\mathbb{1} & \mathbb{1} & \mathbb{1} \\
\mathbb{1} & \mathbb{1} & \mathbb{1}
\end{array}\right] .
$$

Under these assumptions, an optimal control signal $\boldsymbol{u}^{*}(\boldsymbol{x}, t)$ may be computed for system (36) if there is a smooth solution $V(\boldsymbol{x}, t)$, with $V\left(\boldsymbol{x}_{0}, t\right) \equiv 0$ for $t \geq 0$, to the following HJBI equation [27]: 


$$
\begin{aligned}
\frac{\partial V}{\partial t} & +\frac{\partial^{\prime} V}{\partial \boldsymbol{x}} f(\boldsymbol{x}, t) \\
& +\frac{1}{2} \frac{\partial^{\prime} V}{\partial \boldsymbol{x}}\left[\frac{1}{\gamma^{2}} k(\boldsymbol{x}, t) k^{\prime}(\boldsymbol{x}, t)-g(\boldsymbol{x}, t) \boldsymbol{R}^{-1} g^{\prime}(\boldsymbol{x}, t)\right] \frac{\partial V}{\partial \boldsymbol{x}} \\
& -\frac{\partial^{\prime} V}{\partial \boldsymbol{x}} g(\boldsymbol{x}, t) \boldsymbol{R}^{-1} \boldsymbol{S}^{\prime} h(\boldsymbol{x})+\frac{1}{2} h^{\prime}(\boldsymbol{x})\left(\boldsymbol{Q}-\boldsymbol{S} \boldsymbol{R}^{-1} \boldsymbol{S}^{\prime}\right) h(\boldsymbol{x})=0
\end{aligned}
$$

for each $\gamma>\sqrt{\sigma_{\max }(\boldsymbol{R})} \geq 0$, where $\sigma_{\max }$ stands for the maximum singular value. In such a case, the optimal state feedback control law is derived as follows [10]:

$$
\boldsymbol{u}^{*}=-\boldsymbol{R}^{-1}\left(\boldsymbol{S}^{\prime} h(\boldsymbol{x})+g^{\prime}(\boldsymbol{x}, t) \frac{\partial V(\boldsymbol{x}, t)}{\partial \boldsymbol{x}}\right)
$$

As stated before, the solution of the HJBI equation depends on the choice of the cost variable, $\zeta$, and particularly on the selection of function $h(\tilde{\boldsymbol{x}})$ (see (33)). In this paper, this function is taken to be equal to the error vector, i.e., $h(\tilde{\boldsymbol{x}})=\tilde{\boldsymbol{x}}$. Once this function has been selected, computing the control law $\boldsymbol{u}$ will require finding the Lyapunov function, $V(\tilde{\boldsymbol{x}}, t)$, to the HJBI equation posed in (38). Refer to [24] for more details about how to find the candidate Lyapunov function.

The following theorem will help to do this.

Theorem 1[24]. Let $V\left(x_{\eta}, t\right)$ be the scalar function:

$V\left(\boldsymbol{x}_{\eta}, t\right)=\frac{1}{2} \boldsymbol{x}_{\boldsymbol{\eta}}^{\prime} \boldsymbol{T}_{\boldsymbol{o}}^{\prime}\left[\begin{array}{ccc}\mathscr{J}(\boldsymbol{\eta}) & \mathbb{1} & \mathbb{1} \\ \mathbb{O} & \boldsymbol{Y} & \boldsymbol{X}-\boldsymbol{Y} \\ \mathbb{D} & \boldsymbol{X}-\boldsymbol{Y} & \boldsymbol{Z}+\boldsymbol{Y}\end{array}\right] \boldsymbol{T}_{\boldsymbol{o}} \boldsymbol{x}_{\eta}$,

where $\boldsymbol{X}, \quad \boldsymbol{Y}$ and $\boldsymbol{Z} \in \mathfrak{R}^{n \times n}$ are constant, symmetric, and positive definite matrices such that $\boldsymbol{Z}-\boldsymbol{X} \boldsymbol{Y}^{-1} \boldsymbol{X}+2 \boldsymbol{X}>\mathbb{O}$, and $\boldsymbol{T}_{\mathbf{0}}$ is as defined in (37). Let $\boldsymbol{T}$ be the matrix appearing in (32). If these matrices verify the following equation:

$$
\begin{aligned}
& {\left[\begin{array}{ccc}
\mathbb{1} & \boldsymbol{Y} & \boldsymbol{X} \\
\boldsymbol{Y} & 2 \boldsymbol{X} & \boldsymbol{Z}+2 \boldsymbol{X} \\
\boldsymbol{X} & \boldsymbol{Z}+2 \boldsymbol{X} & \mathbb{1}
\end{array}\right]+\boldsymbol{Q}+\frac{1}{\gamma^{2}} \boldsymbol{T}^{\prime} \boldsymbol{T}} \\
& \quad-\left(\boldsymbol{S}^{\prime}+\boldsymbol{T}\right)^{\prime} \boldsymbol{R}^{-1}\left(\boldsymbol{S}^{\prime}+\boldsymbol{T}\right)=\mathbb{O},
\end{aligned}
$$

then, function $V\left(x_{\eta}, t\right)$ constitutes a solution to the HJBI, for a sufficiently high value of $\gamma$.

Proof 1. The proof of this theorem is obtained following the steps presented in [22].

Matrix $\boldsymbol{T}=\left[\begin{array}{lll}\boldsymbol{T}_{\mathbf{1}} & \boldsymbol{T}_{\mathbf{2}} & \boldsymbol{T}_{3}\end{array}\right]$ can be computed by solving the Riccati algebraic equations from (41).

Once matrix $\boldsymbol{T}$ is computed, by substituting $V(\tilde{\boldsymbol{x}}, t)$ into (39), the control law $\boldsymbol{u}^{*}$ corresponding to the $\mathscr{H}_{\infty}$ optimal index $\gamma$ is given by:

$$
u^{*}=-R^{-1}\left(S^{\prime}+T\right) \tilde{x}
$$

Finally, if the control law (42) is placed into (31), and taking into account the error vector (30) and matrix $T$ obtained from (41), the optimal control law can be written in a compact form as follows:

$$
\begin{aligned}
\tau_{\eta_{a}}^{*}= & M(\eta) \ddot{\eta}_{d}+C(\eta, \dot{\eta}) \dot{\eta} \\
& -M(\boldsymbol{\eta})\left(\boldsymbol{K}_{D} \dot{\tilde{\eta}}+\boldsymbol{K}_{P} \tilde{\eta}-K_{I} \int \tilde{\eta} d t\right)
\end{aligned}
$$

where:

$$
\begin{aligned}
& \boldsymbol{K}_{\boldsymbol{D}}=\boldsymbol{T}_{1}^{-1}\left(\boldsymbol{T}_{2}+\boldsymbol{M}(\boldsymbol{\eta})^{-1} \boldsymbol{C}(\boldsymbol{\eta}, \dot{\boldsymbol{\eta}}) \boldsymbol{\eta}_{1}+\boldsymbol{M}(\boldsymbol{\eta})^{-1} \boldsymbol{R}^{-1}\left(\boldsymbol{S}_{1}^{\prime}+\boldsymbol{T}_{1}\right)\right), \\
& \boldsymbol{K}_{\boldsymbol{P}}=\boldsymbol{T}_{1}^{-1}\left(\boldsymbol{T}_{3}+\boldsymbol{M}(\boldsymbol{\eta})^{-1} \boldsymbol{C}(\boldsymbol{\eta}, \dot{\boldsymbol{\eta}}) \boldsymbol{T}_{2}+\boldsymbol{M}(\boldsymbol{\eta})^{-1} \boldsymbol{R}^{-1}\left(\boldsymbol{S}_{2}^{\prime}+\boldsymbol{T}_{2}\right)\right), \\
& \boldsymbol{K}_{\boldsymbol{I}}=\boldsymbol{T}_{1}^{-1}\left(\boldsymbol{M}(\boldsymbol{\eta})^{-1} \boldsymbol{C}(\boldsymbol{\eta}, \dot{\boldsymbol{\eta}}) \boldsymbol{T}_{3}+\boldsymbol{M}(\boldsymbol{\eta})^{-1} \boldsymbol{R}^{-1}\left(\boldsymbol{S}_{3}^{\prime}+\boldsymbol{T}_{3}\right)\right) .
\end{aligned}
$$

A particular case can be obtained when the components of weighting compound $\mathbf{W}^{\prime} \mathbf{W}$ verify:

$$
\begin{aligned}
& \boldsymbol{Q}_{1}=\omega_{1}^{2} \mathbb{1}, \quad \boldsymbol{Q}_{2}=\omega_{2}^{2} \mathbb{1}, \quad \boldsymbol{Q}_{3}=\omega_{3}^{2} \mathbb{1}, \quad \boldsymbol{R}=\omega_{u}^{2} \mathbb{1}, \\
& \boldsymbol{Q}_{12}=\boldsymbol{Q}_{13}=\boldsymbol{Q}_{23}=\mathbb{D}, \quad \boldsymbol{S}_{1}=\boldsymbol{S}_{2}=\boldsymbol{S}_{3}=\mathbb{D} .
\end{aligned}
$$

In this case, the following analytical expressions for the gain matrices have been obtained:

$\boldsymbol{K}_{\boldsymbol{D}}=\frac{\sqrt{\omega_{2}^{2}+2 \omega_{1} \omega_{3}}}{\omega_{1}} \mathbb{1}+\boldsymbol{M}(\boldsymbol{\eta})^{-1}\left(\boldsymbol{C}(\boldsymbol{\eta}, \dot{\boldsymbol{\eta}})+\frac{1}{\omega_{u}^{2}} \mathbb{1}\right)$

$\boldsymbol{K}_{\boldsymbol{P}}=\frac{\omega_{3}}{\omega_{1}} \mathbb{1}+\frac{\sqrt{\omega_{2}^{2}+2 \omega_{1} \omega_{3}}}{\omega_{1}} \boldsymbol{M}(\boldsymbol{\eta})^{-1}\left(\boldsymbol{C}(\boldsymbol{\eta}, \dot{\boldsymbol{\eta}})+\frac{1}{\omega_{u}^{2}} \mathbb{1}\right)$,

$\boldsymbol{K}_{\boldsymbol{I}}=\frac{\omega_{3}}{\omega_{1}} \boldsymbol{M}(\boldsymbol{\eta})^{-1}\left(\boldsymbol{C}(\boldsymbol{\eta}, \dot{\boldsymbol{\eta}})+\frac{1}{\omega_{u}^{2}} \mathbb{1}\right)$.

where the parameters $\omega_{1}, \omega_{2}, \omega_{3}$ and $\omega_{u}$ can be tuned by a systematic procedure keeping in mind a linear PID control action interpretation.

These expressions have an important property: they do not depend on the parameter $\gamma$. So, an algebraic expression for computing the general optimal solution for this particular case is obtained.

\section{SIMULATION RESULTS}

The proposed control strategy, using an integral backstepping controller in cascade with a nonlinear $\mathscr{H}_{\infty}$ controller, named IntBS-NL. $\mathscr{H}_{\infty}$, has been tested by simulation in order to corroborate the effectiveness to solve the path tracking problem when sustained disturbances affect the whole system. Simulations have been performed with a more accurate model as well as saturated control inputs, which emulates a real quadrotor helicopter. This model considers that the axes 
of rotation of the body-fixed frame are parallel to the axes passing through the center of mass, and its origin is displaced by a position $\boldsymbol{r}$ to the center of mass, resulting in crossed inertia terms in the moment of inertia tensor. Moreover, this assumption results in a strongly-coupled dynamic model, with crossed terms in the inertia matrix and in the Coriolis and centrifugal matrix between $\xi$ and $\eta$, and in the gravitational force vector. Taking into account that the simplified model derived in Section II is used just for control synthesis purposes, structural uncertainties are present because that model considers a moment of inertia tensor with only diagonal inertia terms, no crossed inertia terms between $\xi$ and $\eta$, Coriolis and centrifugal terms only on the rotational subsystem, and gravitational terms just on the translational subsystem.

In addition, an amount of $\pm 30 \%$ in the uncertainty of the elements of the moment of inertia tensor and the mass are also considered to test the robustness provided by the control strategies with respect to parametric uncertainty. Finally, sustained disturbances affecting all the degrees of freedom are applied in different instants of time to check the disturbance rejection capability of the proposed controllers.

Furthermore, simulations comparing the control structure developed in this paper ( IntBS-NL. $\mathscr{H}_{\infty}$ ) with the one of [2] (cascade strategy using integral backstepping controllers in both inner and outer loops-IntBS) and that of [23] (cascade strategy using a backstepping controller in the inner loop and a nonlinear $\mathscr{H}_{\infty}$ controller in the outer loop-BS-NL $\mathscr{H}_{\infty}$ ) have been performed in order to show the improvement obtained with the proposed strategy. The first work proposes an integral backstepping approach, which uses the integral term in the first step of the procedure. It has been chosen for the comparison analysis because it is able to present similar performance results, as well as to reject sustained disturbances. The second work uses a backstepping control law in cascade with a nonlinear $\mathscr{H}_{\infty}$ controller to perform path tracking. This strategy has been selected to illustrate that the controller is not able to reject sustained disturbances without the use of the integral action.

The values of the model parameters used for simulations are as follows: $m=0.74 \mathrm{~kg}, l=0.21 \mathrm{~m}, g=9.81 \mathrm{~m} / \mathrm{s}^{2}$ and $I_{x x}=I_{y y}=0.004 \mathrm{kgm}^{2}, I_{z z}=0.0084 \mathrm{kgm}^{2}$. Assuming that the quadrotor helicopter needs, under ideal conditions, a thrust value of about $U_{1} \approx 7.23 \mathrm{~N}$ to perform hovering flight, the following persistent light gusts of wind are considered as external disturbances on the aerodynamic forces and moments: $A_{x}=1 \mathrm{~N}$ at $t=5 \mathrm{~s} ; A_{p}=1 \mathrm{Nm}$ at $t=10 \mathrm{~s}$; and $A_{y}=1 \mathrm{~N}$ at $t=15 \mathrm{~s} ; A_{q}=1 \mathrm{Nm}$ at $t=20 \mathrm{~s} ; A_{z}=1 \mathrm{Nm}$ at $t=25 \mathrm{~s}$; and $A_{r}=1 \mathrm{Nm}$ at $t=30 \mathrm{~s}$.

For the translational motion, the same parameters were adjusted for all backstepping controllers as follows: $c_{1_{x}}=c_{1_{y}}=c_{1_{z}}=17, c_{2 x}=c_{2_{y}}=c_{2_{z}}=2.5, k_{\xi_{x}}=k_{\xi_{y}}=k_{\xi_{z}}=1.5$. The nonlinear $\mathscr{H}_{\infty}$ controller gains were tuned with the following values: $\omega_{1}=0.1, \omega_{2}=3, \omega_{3}=9$ and $\omega_{u}=1.5$.

The first reference path used is a circle evolving in the $\mathfrak{R}^{3}$ Cartesian space defined by:

$$
\begin{aligned}
& x_{d}=\frac{1}{2} \cos \left(\frac{\pi t}{20}\right), \quad y_{d}=\frac{1}{2} \sin \left(\frac{\pi t}{20}\right), \\
& z_{d}=2.5-2 \cos \left(\frac{\pi t}{20}\right), \quad \psi_{d}=0 .
\end{aligned}
$$

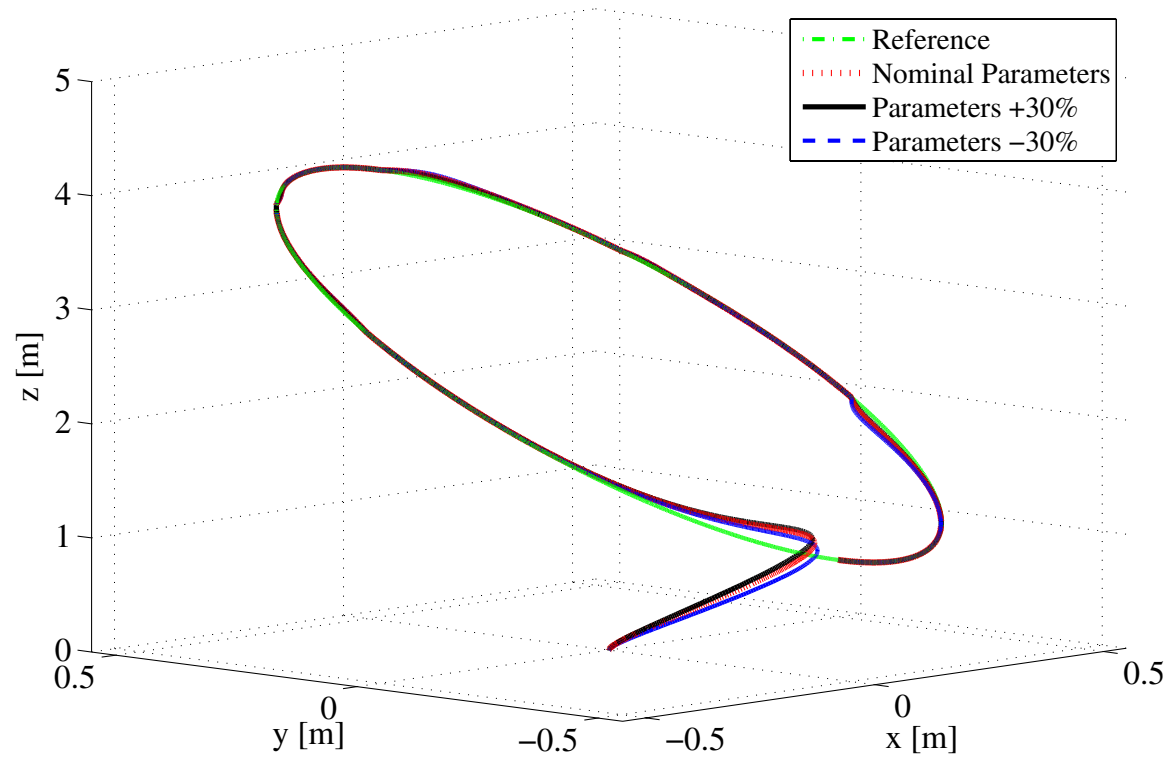

Fig. 3. Path tracking. 

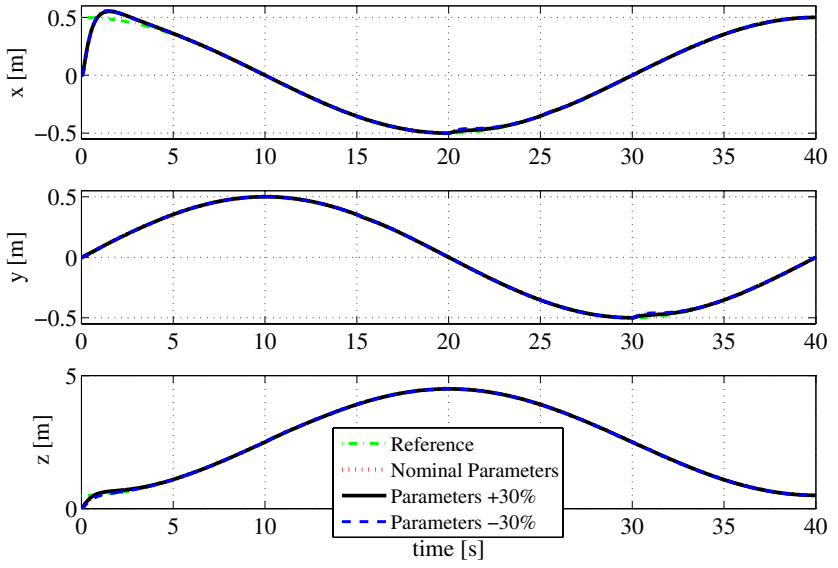

Fig. 4. Position $(x, y, z)$.

For this trajectory, the initial conditions of the helicopter are $\boldsymbol{\xi}_{0}=\left[\begin{array}{lll}0 & 0 & 0\end{array}\right]^{\prime} \mathrm{m}$ and $\boldsymbol{\eta}_{0}=\left[\begin{array}{lll}0 & 0 & 0.5\end{array}\right]^{\prime} \mathrm{rad}$.

Figs 3 to 6 show the simulation results of the path tracking for the first reference trajectory. They illustrate how, starting from an initial position far from the reference, the proposed control strategy is able to make the quadrotor helicopter follow the reference trajectory.

Figs 4 and 5 show the position and position errors. It can be seen that zero steady-state error is achieved for all coordinates, even if structural uncertainty and different model parameter values are considered in the vehicle. Moreover, the translational controller provides the quadrotor helicopter with a fast and smooth recovery to the reference trajectory when external disturbances affect it.

The behaviour produced by the nonlinear $\mathscr{H}_{\infty}$ control law in the rotational motion is shown in Fig. 6. It can be observed how that controller reacts when the helicopter is disturbed on the six degrees of freedom. When disturbances affect the translational motion, the rotational controller counter-attack them so quickly that the damages is minimized for the inner-loop. The first two graphs show how the references generated by the integral backstepping controller (translational motion loop), i.e., $\phi_{d}$ and $\theta_{d}$, varies in its attainment of an appropriate performance in the translational loop. It is due to the system coupling.

A second simulation collection has been obtained with a vertical helix reference trajectory. For this path, the helicopter starts at the initial position $\xi_{0}=\left[\begin{array}{lll}0.5 & 0 & 1.0\end{array}\right]^{\prime} \mathrm{m}$ and $\eta_{0}=\left[\begin{array}{lll}0 & 0 & 0.5\end{array}\right]^{\prime} \mathrm{rad}$, with an amount of $+30 \%$ of uncertainty in the elements of the mass and the inertia matrix. In these simulations, results attained by the integral backstepping with the nonlinear $\mathscr{H}_{\infty}$ control structure are compared with the ones achieved by the IntBS. A third control strategy, the BS-NL. $\mathscr{H}_{\infty}$, is also performed for the comparison analysis.

The parameters for all control structures have been adjusted to obtain a smooth reference tracking, with a quick
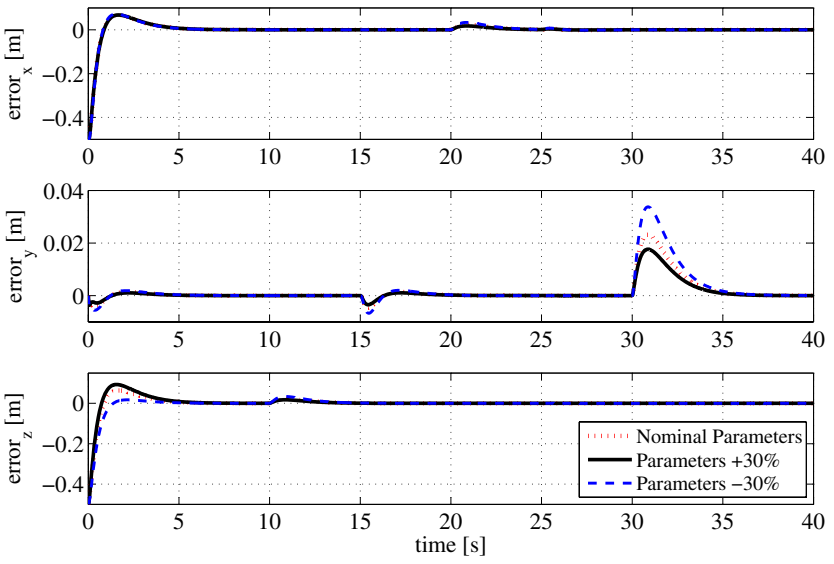

Fig. 5. Position error $(x, y, z)$.
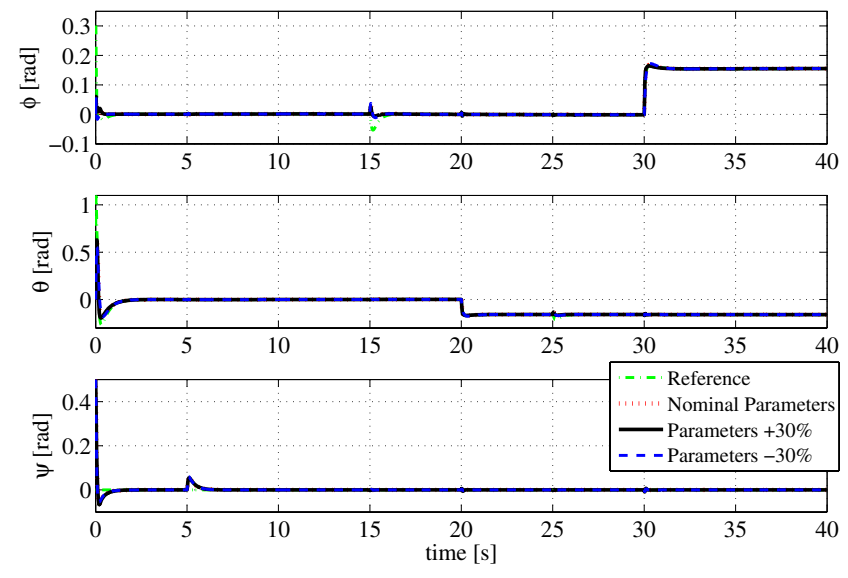

Fig. 6. Orientation $(\varphi, \theta, \psi)$.

disturbance rejection, when it is possible, and a small transient error. As commented before, the backstepping parameters are the same for all translational controllers. The parameters for the rotational controller used in the IntBS structure have been synthesized to produce a similar behaviour of the nonlinear $\mathscr{H}_{\infty}$ rotational controller. The simulations results are depicted in Figs 7 to 10 .

These figures illustrate that the proposed control strategy and the IntBS present a robust path tracking when sustained disturbances are applied on the whole quadrotor helicopter degrees of freedom. Despite of the faster time response of the translational controllers of both IntBS-NL $\mathscr{H}_{\infty}$ and IntBS control strategies, it can be clearly observed in Fig. 8 that the translational motion response, in the case of the IntBS, converges slower to the reference than the proposed IntBS-NL. $\mathscr{H}_{\infty}$ control strategy. As commented in [25], increasing the positive feedbacks gains $k_{\xi_{i}}, c_{1_{i}}$ and $c_{2_{i}}$ will eventually give stability and convergence, for the backstepping procedure with the integral term in the first step, when $g_{1}\left(\boldsymbol{x}_{\mathbf{1}}\right)$ is constant (see (7)), which is the case for the quadrotor helicopter model. 


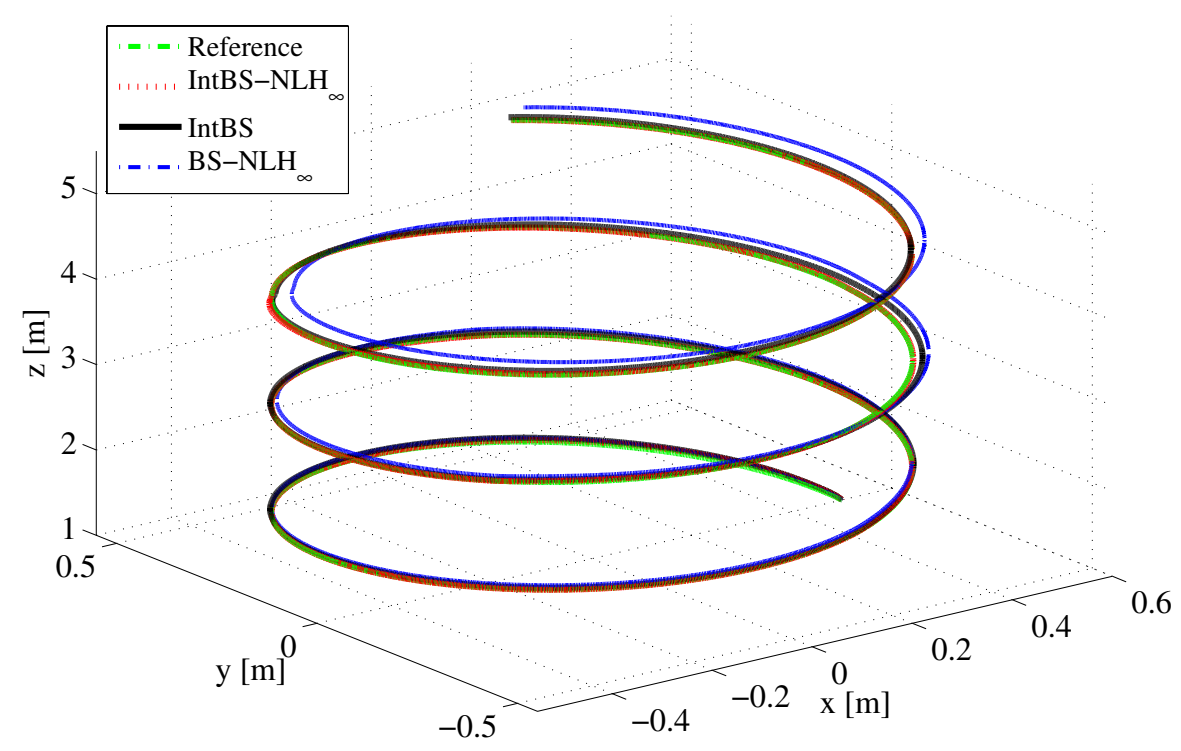

Fig. 7. Path following.
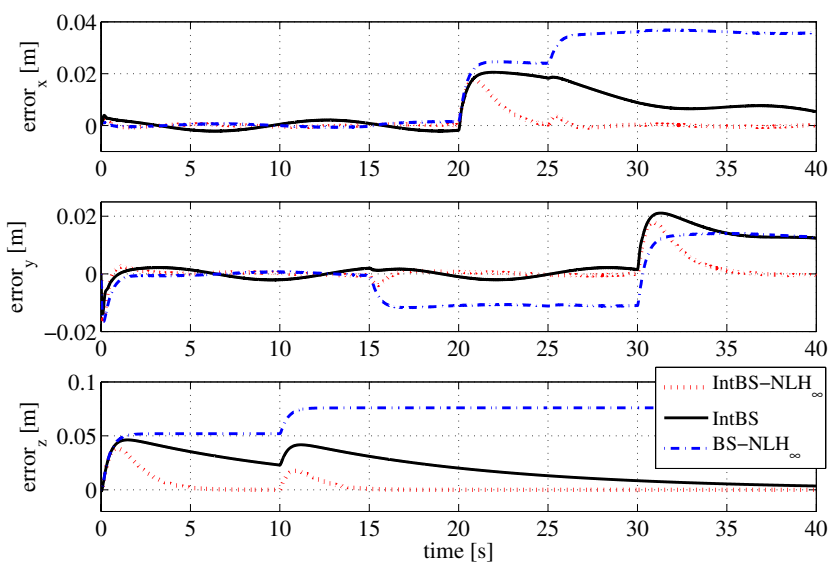

Fig. 8. Position error $(x, y, z)$.

When the control strategy proposed in this paper is compared with the one gives by [23], the improvement generated by the use of the integral action is clearly pointed out. Besides, to highlight this feature, the $\omega_{3}$ parameter of the nonlinear $\mathscr{H}_{\infty}$ controller is settled null in the second control strategy. It can be clearly observed that, in the BS-NL. $\mathscr{H}_{\infty}$ case, the vehicle leaves the reference trajectory when the disturbances are introduced, and it never reaches the reference again. In addition, Figs 8 and 9 show that the helicopter degrees of freedom can not obtain a null steady-state when no integral term is considered in the controllers synthesis. To corroborate the fact that null steady-state is achieved for the proposed control strategy in this paper, Table I presents the final errors in the $3 \mathrm{D}$ space.

Furthermore, the integral square error (ISE) performance indexes obtained from the simulation results are
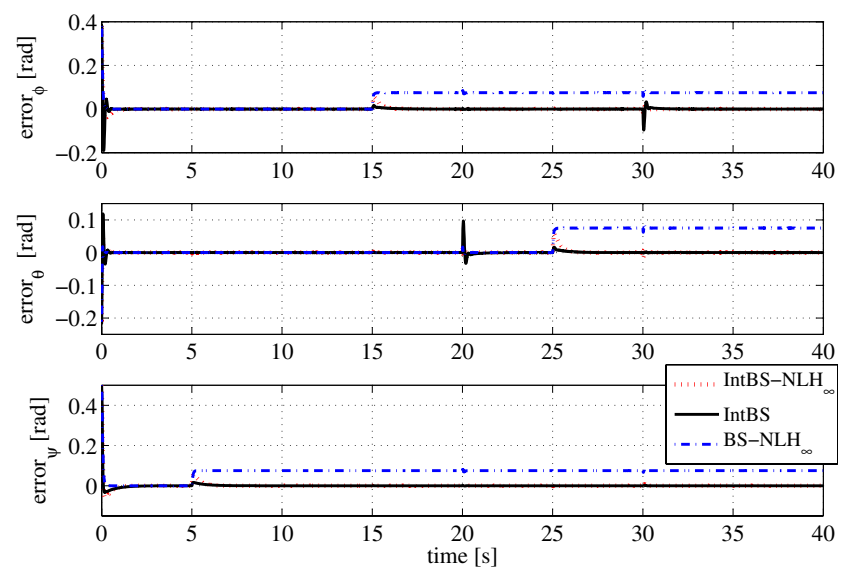

Fig. 9. Orientation $(\varphi, \theta, \psi)$.

presented in Table II. It can be observed that the performance is improved by the IntBS-NL $\mathscr{H}_{\infty}$ control strategy for all states, which, compared with the IntBs control strategy, presents an improvement of $x \downarrow 96.62 \%, y \downarrow 94.36 \%, z \downarrow 97.33 \%$, $\phi \downarrow 38.42 \%, \theta \downarrow 51.84 \%$ and $\psi \downarrow 20.39 \%$. These index values corroborate the results presented in Figs 8 and 9.

On the other hand, the integral absolute derivative control signal (IADU) index has been computed for all control signals in the three control strategies (depicted in Fig. 10). This performance index is appropriate to check the control signals' smoothness. Table III shows a comparison between the results provided by the IntBs-NL. $\mathscr{H}_{\infty}$, IntBS and Bs-NL. $\mathscr{H}_{\infty}$ control strategies. In this case, the third strategy presents a smoother control signal. However, as can be seen in Figs 8 and 9, the vehicle presents an offset in steady-state. When the proposed control strategy is compared with the 

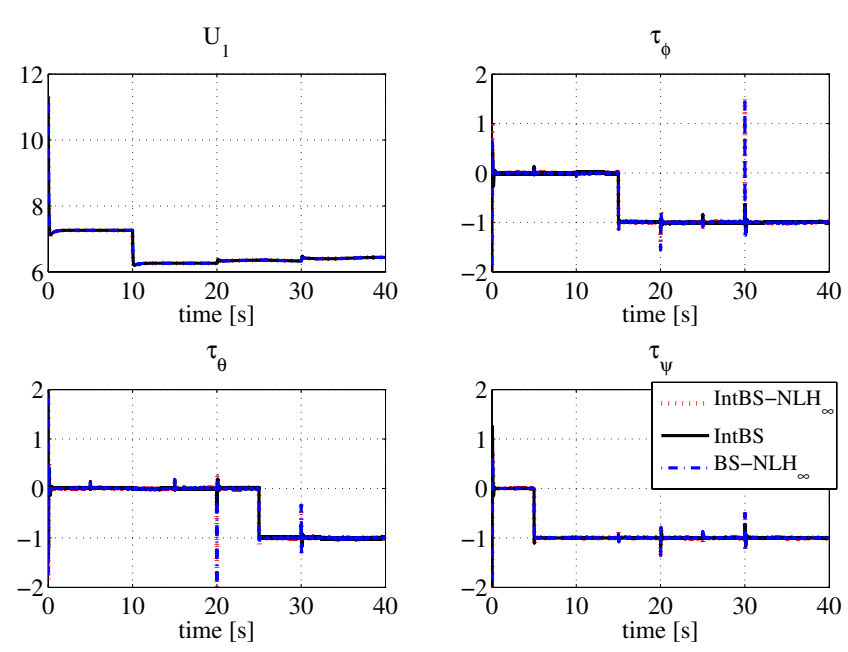

Fig. 10. Control inputs $\left(U_{1}, \tau_{\varphi}, \tau_{\theta}, \tau_{\psi}\right)$.

Table I. XYZ-Axes Final Position Errors.

\begin{tabular}{lccc}
\hline Axis & IntBS-NL. $\mathscr{H}_{\infty}$ & IntBS & BS-NL. $\mathscr{H}_{\infty}$ \\
\hline$x$-axis & $0,000 m$ & $-0,005 m$ & $-0,036 m$ \\
$y$-axis & $0,000 m$ & $-0,012 m$ & $-0,013 m$ \\
$z$-axis & $0,000 m$ & $-0,004 m$ & $-0,076 m$ \\
\hline
\end{tabular}

Table II. ISE Index Performance Analysis.

\begin{tabular}{lcrr}
\hline States & IntBs-NL. $\mathscr{H}_{\infty}$ & IntBs & Bs-NL. $\mathscr{H}_{\infty}$ \\
\hline$x$ & 0.0581 & 1.7184 & 2.6305 \\
$y$ & 0.0757 & 1.3414 & 0.4271 \\
$z$ & 0.3157 & 11.8193 & 22.9256 \\
$\phi$ & 3.3139 & 5.3811 & 20.0234 \\
$\theta$ & 0.7943 & 1.6494 & 10.9833 \\
$\psi$ & 6.3049 & 7.9193 & 29.3531 \\
\hline
\end{tabular}

Table III. IADU Index Performance Analysis.

\begin{tabular}{lcrr}
\hline Control Signals & IntBs-NL. $\mathscr{H}_{\infty}$ & \multicolumn{1}{c}{ IntBs } & Bs-NL. $\mathscr{H}_{\infty}$ \\
\hline$U_{1}$ & 6.1642 & 6.0747 & 6.1033 \\
$\tau_{\phi}$ & 29.8298 & 95.0931 & 28.7654 \\
$\tau_{\theta}$ & 33.0568 & 108.4128 & 31.7607 \\
$\tau_{\psi}$ & 17.5876 & 12.3266 & 16.5672 \\
\hline
\end{tabular}

IntBS one, the index values are almost the same for the control input $U_{1}$, although the IntBs-NL $\mathscr{H}_{\infty}$ strategy has provided a faster response than the other one. For the rotational loop, two of three control signals have been obtained with higher smoothness. Again, it is noted that, despite of some smoother signals presented by the others strategies, only the IntBs-NL. $\mathscr{H}_{\infty}$ strategy has achieved the control objectives.

\section{CONCLUSIONS}

In this paper a robust control strategy to solve the path tracking problem for a quadrotor helicopter has been presented. This control structure has been designed in consideration of external disturbances, like aerodynamic forces and moments, acting on all degrees of freedom, parametric uncertainties and unmodeled dynamics.

The proposed control strategy combines an integral backstepping approach to control the translational movements with a nonlinear $\mathscr{H}_{\infty}$ controller designated to stabilize the helicopter. The translational controller has been designed through an integral backstepping procedure, using the integral term in its second step. This controller guarantees stability and convergence of the tracking error for a generic plant when a maintained disturbance affect the system and the reference signal is time-varying. A comparison with other integral backstepping controllers using the integral term in the first step has been provided, and it has confirmed the improvement of the approach used in this paper.

On the other hand, a robust control based on nonlinear $\mathscr{H}_{\infty}$ theory has been used for the helicopter stabilization, which is able to reject sustained disturbances.

The robustness of the control strategy have been observed when parametric and structural uncertainties have been considered during the simulations. Simulations results have been presented to corroborate an appropriate performance obtained to solve the path tracking problem, where the use of integral action in the inner and outer loop controllers has provided the capability to deal with sustained disturbances when all degrees of freedom are affected by this kind of perturbation in different moments of time.

Finally, to show the improvements attained by the proposed control strategy, a comparative analysis among the proposed control strategy and others recent controllers has been carried out by means of the ISE and IADU performance indexes.

\section{REFERENCES}

1. Bouabdallah, S. and R. Siegwart, "Design and control of a miniature quadrotor," In K. Valavanis (Ed.) Advances in unmanned aerial vehicles, volume 33 of Intelligent systems, control and automation: Science and engineering, pp. 171-210. Springer Netherlands (2007).

2. Bouabdallah, S. and R. Siegwart, "Full control of a quadrotor," In Proc. Intell. Robots Sys. IROS 2007, pp. 153-158, San Diego, USA (2007).

3. Cai, G., B. Wang, B. M. Chen, and T. H. Lee, "Design and implementation of a flight control system for an unmanned rotorcraft using rpt control approach," Asian J. Control, Vol. 15, No. 1, pp. 95-119 (2013). 
4. Castillo, P., R. Lozano, and A. Dzul, "Stabilization of a mini rotorcraft with four rotors," IEEE Control Syst. Mag., Vol. 22, No. 6, pp. 45-55 (Dec. 2005).

5. Castillo, P., R. Lozano, and A. E. Dzul, Modelling and Control of Mini-Flying Machines, Springer-Verlag, London, UK (2005).

6. Chen, M. and M. Huzmezan, "A combined MBPC/ 2DOF $\mathscr{H}_{\infty}$ controller for a quad rotor UAV," In Proc. AIAA Guidance Navigation Control Conf. Exhibit, Austin, TX, USA (2003).

7. Colorado, J., A. Barrientos, A. Martinez, B. Lafaverges, and J. Valente, "Mini-quadrotor attitude control based on hybrid backstepping amp; frenet-serret theory," In 2010 IEEE Int. Conf. Robot. Autom. (ICRA), Anchorage, Alaska, pp. 1617-1622 (2010).

8. Das, A., F. Lewis, and K. Subbarao, "Backstepping approach for controlling a quadrotor using lagrange form dynamics," J. Intell. Robot. Syst., Vol. 56, No. 1-2, pp. 127-151 (2009).

9. Fantoni, I. and R. Lozano, Non-linear Control for Underactuated Mechanical Systems, Springer-Verlag, London, UK (2002).

10. Feng, W. and I. Postlethwaite, "Robust nonlinear $H_{\infty} /$ adaptative control of robot manipulator motion," Proc. Instn. Mech. Engrs., Vol. 208, pp. 221-230 (1994).

11. Guenard, N., T. Hamel, and R. Mahony, "A practical visual servo control for an unmanned aerial vehicle," IEEE Trans. Robot., Vol. 24, No. 2, pp. 331-340 (2008).

12. Hoffmann, G. M., H. Huang, S. L. Waslander, and C. J. Tomlin, "Quadrotor helicopter flight dynamics and control: Theory and experiment," In Proc. AIAA Guidance Navigation Control Conf. Exhibit, pp. 1-20, Hilton Head, SC, USA (2007).

13. Kanellakopoulos, I. and P. Krein, "Integral-action nonlinear control of induction motors," In Proc. 12th IFAC World Congr., pp. 251-254, Sydney, Australia (1993).

14. Krstic, M., P. V. Kokotovic, and I. Kanellakopoulos, Nonlinear and Adaptive Control Design, John Wiley \& Sons, Inc., New York, USA (1995).

15. Madani, T. and A. Benallegue, "Sliding mode observer and backstepping control for a quadrotor unmanned aerial vehicles," In Proc. Amer. Control Conf. 2007. ACC '07, pp. 5887-5892, New York, USA (July, 2007).

16. Mahony, R. and T. Hamel, "Robust trajectory tracking for a scale model autonomous helicopter," Int. J. Robust Nonlinear Control, Vol. 14, pp. 1035-1059 (2004).

17. Mederreg, L., F. Diaz, and N. K. M'Sirdi, "Nonlinear backstepping control with observer design for a 4 rotors helicopter," In Proc. IFAC Int. Conf. Advances Vehicle Control Safety (AVXS'04), pp. 24-31 (2003).

18. Mian, A. A. and W. Daobo, "Modeling and backsteppingbased nonlinear control strategy for a $6\{\mathrm{DOF}\}$ quadrotor helicopter," Chin. J. Aeronaut., Vol. 21, No. 3, pp. 261268 (2008).

19. Mistler, V., A. Benallegue, and N. K. M'Sirdi, "Exact linearization and noninteracting control of a 4 rotors helicopter via dynamic feedback," In Proc. IEEE Int. Workshop Robot Human Inter. Communic., pp. 586-593 (2001).

20. Mokhtari, A., A. Benallegue, and Y. Orlov, "Exact linearization and sliding mode observer for a quadrotor unmanned aerial vehicle," Int. J. Robot. Autom., Vol. 21, No. 1, pp. 39-49 (2006).

21. Önder Efe, M., "Battery power loss compensated fractional order sliding mode control of a quadrotor uav," Asian J. Control, Vol. 14, No. 2, pp. 413-425 (2012).

22. Ortega, M. G., M. Vargas, C. Vivas, and F. R. Rubio, "Robustness improvement of a nonlinear $H_{\infty}$ controller for robot manipulators via saturation functions," $J$. Robot. Syst., Vol. 22, No. 8, pp. 421-437 (2005).

23. Raffo, G. V., M. G. Ortega, and F. R. Rubio, "Backstepping/nonlinear $\mathscr{H}_{\infty}$ control for path tracking of a quadrotor unmanned aerial vehicle," In Proc. 2008 Amer. Control Conf. ACC2008, pp. 3356-3361, Seattle, WA, USA (July, 2008).

24. Raffo, G. V., M. G. Ortega, and F. R. Rubio, "An integral predictive/nonlinear $\mathscr{H}_{b_{0}}$ control structure for a quadrotor helicopter," Automatica, Vol. 46, pp. 29-39 (2010).

25. Skjetne, R. and T. I. Fossen, "On integral control in backstepping: Analysis of different techniques," In Proc. 45th IEEE Conf. Decis. Control, pp. 1899-1904, Boston, MA, USA (2004).

26. Spong, M. W., S. Hutchinson, and M. Vidyasagar, Robot Modeling and Control, John Wiley \& Sons, Inc, New York (2006).

27. van der Schaft, $\mathrm{A}$., $\mathrm{L}_{2}$-Gain and Passivity Techniques in Nonlinear Control, Springer-Verlag, New York (2000). 2nd Ed.

28. Xu, R. and Ü. Özgüner, "Sliding mode control of a class of underactuated systems," Automatica, Vol. 44, No. 1, pp. 233-241 (2008).

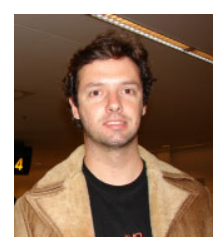

Guilherme V. Raffo was born in Porto Alegre, Brazil. He received the B.Sc. degree in automation and control engineering from the Pontifical Catholic University of Rio Grande do Sul, Brazil, in 2002, his specialist degree in industrial automation from the Federal University of Rio Grande do Sul, Brazil, in 2003, his M.Sc. degree in electrical engineering from the Federal University of Santa Catarina, Brazil, in 2005, his M.Sc. degree in automation, robotics and telematic in 2007 and his Ph.D. degree in 2011, both from the University of Seville, Spain, and was a postdoctoral fellow in 2011-2012 at the Federal University of Santa Catarina, Brazil. He is 
currently Adjunct Professor at Federal University of Minas Gerais, Brazil. He is the author or coauthor of more than 30 publications including journal papers, book chapters, and conference proceedings. His current research interests include autonomous vehicles, robust control, nonlinear control, $\mathrm{H}_{\infty}$ control theory, predictive control, time-delay systems, robotics, and underactuated systems.

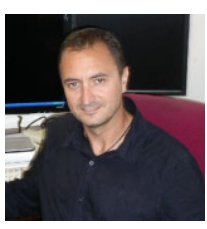

Manuel G. Ortega was born in Jaén, Spain. He received the M.Sc. degree in Industrial Electrical Engineering, and the Ph.D. degree in System Engineering and Automation (with doctoral award), both from the University of Seville, Spain, in 1995 and 2001, respectively. From 1996 to 2004, he was Research Assistant under several grants and Assistant Professor at the Department of Systems Engineering and Automation of the University of Seville. Since 2004, he has been Associate Professor at the same Department. Dr. Ortega has worked on several research and development projects in cooperation with industry. He is the author or coauthor of more than 60 publications including journal papers, book chapters, and conference proceedings. His current research interests include robust control, $\mathrm{H}_{\infty}$ control theory, nonlinear control systems, robotics, and process control.

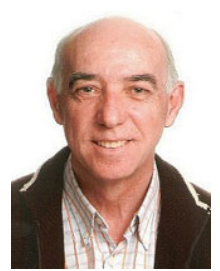

Francisco R. Rubio received the Industrial Electrical Engineering degree and Doctorate from the Escuela Técnica Superior de Ingenieros Industriales de Sevilla in 1981 and 1985, respectively. He received the CITEMA award for the best work on automation by a young engineer in 1980 . He is Professor in the Department of Systems Engineering and Automatic Control of the University of Seville. He has worked on various research and development projects in cooperation with industry. His current interests are in the areas of adaptive control, robust process control, nonlinear control systems, robotics and networked control systems. He has written three books: Advanced Control of Solar Plants and Control of Solar Energy Systems published by SpringerVerlag and Control Adaptativo y Robusto published by Seville University. He has authored and co-authored more than 200 technical papers published in international journals and conference proceedings. 